\title{
Porous Anodic Alumina Membrane as a Sample Support for MALDI-TOF MS Analysis of Salt-Containing Proteins
}

\author{
Yuebo Wang* and Xinghua Xia \\ Key Laboratory of Analytical Chemistry for Life Sciences, Department of Chemistry, Nanjing University, \\ Nanjing, People's Republic of China \\ Yinlong Guo \\ Shanghai Mass Spectrometry Center, Shanghai Institute of Organic Chemistry, Chinese Academy of Science, \\ Shanghai, People's Republic of China
}

Matrix-assisted laser desorption/ionization time-of-flight mass spectrometric (MALDI-TOF MS) analysis of proteins in salt-containing solution was performed for the first time using porous anodic alumina (PAA) membrane as sample support. The resulting spectral quality of proteins under standard sample preparation conditions was superior to that of normal metal sample stages. Analysis of phosphate-doped protein solutions indicated that porous anodic alumina membranes as a target yielded better results than a metallic target for salt-containing solutions. Because of the biocompatibility of the PAA, proteins can be adsorbed on the PAA and thus a washing process can be introduced to remove the salts from the PAA target before MS analysis. This desalting step significantly enhanced spectral quality, and better signal-tonoise ratios were obtained. The present technique is promising for proteomics research. (J Am Soc Mass Spectrom 2005, 16, 1488-1492) (C) 2005 American Society for Mass Spectrometry

S ince the pioneering work by Karas and Hillenkamp, matrix-assisted laser desorption/ionization mass spectrometry (MALDI-MS) has been proven to be a powerfully widespread technique in analytical chemistry attributable to its high capacity to rapidly analyze minute quantities of materials [1], and a rapid and convenient method for the characterization of peptides and proteins from biological samples [2-4] because of its compatibility with biological buffers or other interferences [5, 6]. However, MALDI-MS is not immune to interferences in complex biological samples [7]. Smaller amounts of salts usually results in formation of adduct on the molecular ions of analytes and thus produce broadened and poorly resolved peaks [8]. Since adducts increase peak width and decrease resolution, desalting process before MALDI analysis has been used to increase mass accuracy and spectral qual-

Published online July 14, 2005

Address reprint requests to Dr. Y. Guo, Shanghai Mass Spectrometry Center, Shanghai Institute of Organic Chemistry, Chinese Academy of Science, Shanghai 200032, People's Republic of China. E-mail: ylguo@mail.sioc.ac.cn. Requests can also be addressed to Prof. X. H. Xia, Key Laboratory of Analytical Chemistry for Life Science, Department of Chemistry, Nanjing University, Nanjing 210093, People's Republic of China. E-mail: xhxia@nju.edu.cn.

* Also affiliated with the Shanghai Mass Spectrometry Center, Shanghai Institute of Organic Chemistry, Chinese Academy of Science, Shanghai, People's Republic of China. ity [9]. The removal of the interfering species such as salts and buffers from protein samples, metallic supports modified with functionalized self-assembled layers, $[7,10,11]$ or other types of supports with highly binding affinity for proteins have been suggested [1219]. The other types of supports are nitrocellulose [12], poly(vinylidine difluoride) [15, 16], polyethylene [17], nylon [18], and polyurethane [14, 19].

Porous anodic alumina (PAA) membrane is an inorganic material with self-ordered nanoporous pores. It has a packed array of columnar hexagonal cells with central, uniformly sized holes ranging from 4 to $200 \mathrm{~nm}$ in diameter, pore density as high as $10^{8}$ to $10^{11}$ pores / $\mathrm{cm}^{2}$ [20-22]. The PAA has been extensively used in fabricating nanometer-sized tubes, rods, and wires of various materials with a great flexibility [23-26]. Because the PAA membrane has nano-sized pores and enormous pore density, it may be used in dispersing samples in MALDI analysis. In addition, PAA has a good biocompatibility and can adsorb protein via supramolecular interactions [27, 28], which permits a washing step for the removal of contaminants from protein samples. The PAA with high density of pores and biocompatibility might be used in MALDI analysis of proteins for enhancing spectral quality.

Here, we report on the use of porous anodic alumina (PAA) membrane as sample supports for MALDI-TOF 
MS analysis of proteins. MS results showed that the PAA-based spectral qualities were better than those with metal targets. Adsorption of proteins on the PAA was characterized by using infrared spectroscopic technique. The morphology of the protein deposited on the PAA was examined with scanning electron microscopy. Application of the biocompatible PAA film as support allows the introduction of a washing step before MS analysis for removal of salts and buffer components, resulting in an enhanced spectral quality and better signal-to-noise $(\mathrm{S} / \mathrm{N})$ ratios. The present protocol may be used in analysis of complex mixture of proteins.

\section{Experimental}

Myoglobin, cytochrome $c$, and insulin solutions (Sigma, St. Louis, MO) were prepared with a mixture of water and acetonitrile (vol:vol = 70:30). A phosphate-doped lysozyme (Yuanju Biological Co., Shanghai, China) solution was prepared with 70:30 (vol:vol) phosphate buffer and acetonitrile. Polyurethane adhesive (NIPPOLLAN-DC 205, Nippon Polyurethane Industry Co., Ltd., Tokyo, Japan) was diluted with ethyl acetate until colorless for further use. Porous anodic alumina (PAA) (diameter: $13 \mathrm{~mm}$, pore size: $0.1 \mu \mathrm{m}$ ) was obtained from Whatman International Ltd., (Maidstone, England). Saturated solution of $\alpha$-cyano-4-hydroxycinnamic acid (CHCA, Sigma, St. Louis, MO) in a mixture of 70:30 water/acetonitrile (vol:vol) with $0.1 \%$ trifluoroacetic acid (Tedia Company, Inc., Fairfield, $\mathrm{OH}$ ) was used as matrix for MALDI-TOF MS analysis.

The porous alumina membrane was cleaved into small pieces as MALDI targets. After drying at roomtemperature, PAA piece deposited with $1.0 \mu$ l of freshly prepared protein and matrix mixture (1:25, vol/vol) was adhered to a stainless steel target probe (MALDI target) using $1.0 \mu \mathrm{l}$ of polyurethane adhesive solution. For comparison, direct deposition of the same amount of the protein and matrix mixture to a metal target was also performed. When incorporating a washing step, the protein loaded PAA piece was washed with $20 \mu \mathrm{l}$ of 20:80 methanol/water (vol:vol) solution for a few times before addition of the matrix.

MALDI-TOF MS analyses were performed employing delayed extraction in positive ion mode on a time-of-flight mass spectrometer (Voyager-DE STR, Applied Biosystems, Framingham, MA) using an accelerating potential of $25 \mathrm{kV}$. Spectra were obtained using a nitrogen laser (337 $\mathrm{nm}$ ) adjusted to slightly above threshold. The spectra presented in this paper generally represent the sum of 120 laser shots. Transmission FT-IR spectra (128 scans at a resolution of 8 $\mathrm{cm}^{-1}$ ) were recorded on a Tensor 27 Fourier transform infrared spectrometer (Bruker, Karlsruhe, Germany) equipped with a liquid nitrogen cooled DTGS detector. Morphology of the metal and the PAA target deposited with phosphate-doped lysozyme was examined on a scanning electron microscope
(Hitachi SEM-X650, Hitachi Ltd., Tokyo, Japan) at an acceleration voltage of $20 \mathrm{kV}$.

\section{Results and Discussion}

\section{Adsorption of Lysozyme on PAA Membrane}

To understand the biocompatibility of the porous anodic alumina film, we used lysozyme as a model biomolecule to study its adsorption on the PAA membrane with the help of an infrared spectrometer. Spectra of $40 \mu$ l lysozyme $(5 \mathrm{mg} / \mathrm{ml})$ deposited on a PAA film followed by washing 1 to 3 times were collected. All the spectra showed that lysozyme adsorbed on the PAA membrane as indicated by the two-amide adsorption bands at $1540 \mathrm{~cm}^{-1}$ and $1655 \mathrm{~cm}^{-1}$ [29]. A significant decrease in the band intensities of the sample was observed upon washing the first time (Figure 1), indicating that most of the proteins were washed away from the membrane. However, further washing of the sample only decreased the band intensities slightly, demonstrating that only the lysozyme directly interacting with the PAA surface remained on the surface. These results demonstrate that a washing protocol to remove contaminants from complex protein samples can be introduced before MALDI-TOF MS analysis.

\section{MS Analysis of Proteins on a PAA Membrane}

Comparison between the MS spectra of 10 pmol lysozyme with CHCA as matrix on a porous anodic alumina membrane and a metallic target were performed. Both targets gave signals corresponding to a single charged peak and a doubly charged peak (results not shown). Careful examination of the spectra using automatic S/N calculation software of the spectrometer showed that the $\mathrm{S} / \mathrm{N}$ ratio for the proteins deposited on the PAA target was about three times better than that

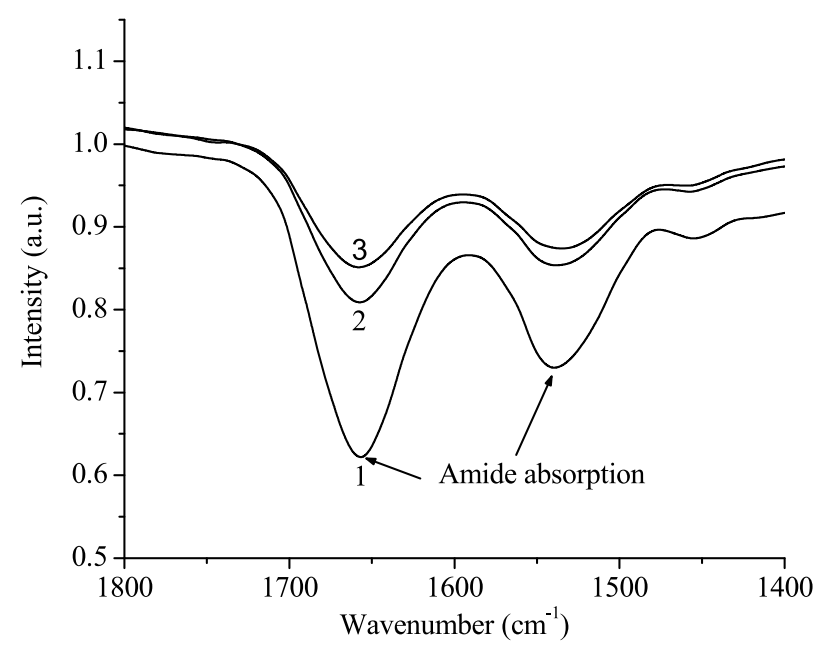

Figure 1. Transmission FT-IR spectra of $40 \mu$ l lysozyme (5 $\mathrm{mg} / \mathrm{ml}$ ) deposited on a PAA membrane (1) and followed by washing with water once (2), and twice (3), respectively. Spectrum of a pure PAA membrane was used as reference. 

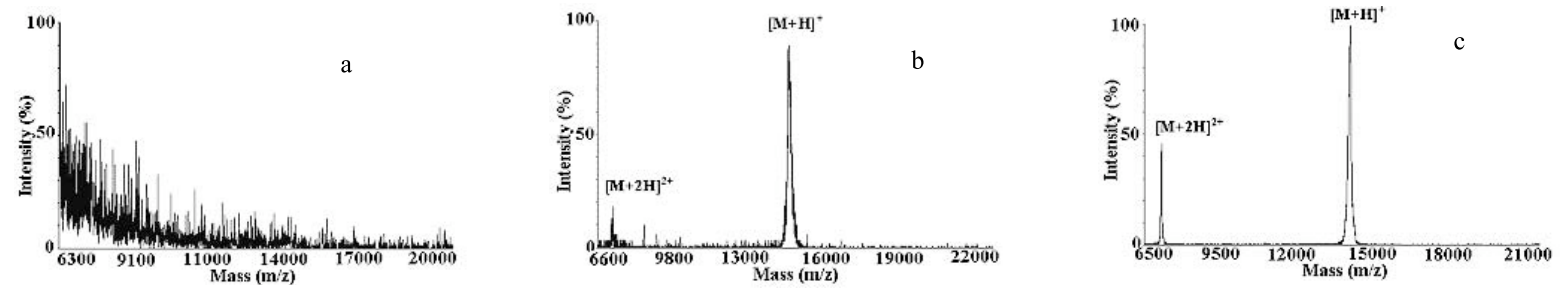

Figure 2. MALDI-TOF mass spectra of lysozyme $(3 \mathrm{mg} / \mathrm{ml})$ in a solution of phosphate buffer and acetonitrile $(7: 3, \mathrm{vol} / \mathrm{vol})$ deposited on a metallic target (a); a PAA target (b). Spectra (c) correspond to the mass spectra of sample $(\mathbf{b})$ washed with methanol/water $(1: 4, \mathrm{vol} / \mathrm{vol})$ three times. CHCA was used as the matrix.

on the metallic target. This could be attributable to the high pore density and good biocompatibility of the PAA membrane that dispersed the protein sample well on its surface, resulting in an enhanced spectral quality and better S/N. The large surface area associated with the pore structure of the PAA membrane allowed the sample to disperse into or out of the pores, also resulting in an increased MS signal. The present PAA target could be applicable to other proteins with similar molecular weight such as myoglobin, cytochrome $c$, and insulin. The $\mathrm{S} / \mathrm{N}$ ratios for myoglobin, cytochrome $c$, and insulin obtained on the PAA targets were 2.86, 2.14, and 2.70 times better than that on the metallic targets, respectively. Average of the six different locations for one sample offered all the results.

\section{Incorporation of a Washing Protocol Before MS Analysis}

Although MALDI-TOF MS is relatively tolerant of impurities such as salts and buffer components, removal of these species will yield higher resolution and mass accuracy. To accomplish this, a desalting step before MS analysis with PAA as support was used for protein analysis with relatively high amounts of salts and buffer components. In our experiments, samples of lysozyme were prepared in $350 \mathrm{mM}$ phosphate and acetonitrile (7:3, $\mathrm{vol} / \mathrm{vol})$. As expected, at significantly higher concentrations of phosphate, no sample signal was observed on a metal target (Figure 2a). In contrast, a weaker signal clearly appeared for a PAA target (Figure $2 \mathrm{~b}$ ), although the peak was wide and the $\mathrm{S} / \mathrm{N}$ ratio was low because of the salt adduct of the analyte molecular ions. The S/N ratio was 6.0 for the phosphate-doped lysozyme deposited on the PAA target. An overall improvement in peak shape was achieved with successive washing steps; peaks became narrower after washing three times (Figure 2c). The reason for the improvement in the peak shape must be due to a decrease in the salt adduct for the molecular ions of the analyte after washing. This result implied that the proton adduct of the molecular ions of the analyte was in the majority. In addition, the intensity of the doubly charged peaks increased and the $\mathrm{S} / \mathrm{N}$ ratio was better than that without washing. The calculated $\mathrm{S} / \mathrm{N}$ ratios were $28.8,89.2$, and 479.1 for washing the phosphatedoped lysozyme deposited onto the PAA target once, twice, and three times, respectively. This washing step could also be applied to the phosphate-doped cytochrome c. As expected, the results were similar to lysozyme. The calculated S/N ratios were 584.6, 625.4, and 988.8 for washing the phosphate-doped cytochrome $c$ deposited onto the PAA target once, twice, and three times, respectively. Obviously, introduction of a washing step before the MS analysis could significantly increase the mass spectral quality of the salt-containing proteins.

SEM imaging of the morphology of the supports deposited with protein was also carried out to understand the improved MS spectral quality. For a metallic target (Figure 3a), a phosphate salt layer was clearly visible besides some branched salt crystals. The protein molecules and matrix were imbedded in the salt layer. The salt layer will shield or scatter the laser beam, resulting in inefficient ionization of the proteins. So, no sample signal was observed on a metal target for a phosphate-doped protein solution (Figure 2a). For a PAA target (Figure $3 b$ ), proteins, matrix, and salt dispersed on the surface and only discontinued salt blocks appeared as indicated by arrows. The salt blocks would shield or scatter the laser beam, resulting in inefficient ionization of the proteins beneath them. However, the proteins exposed on the PAA surface could adsorb laser beam for ionization. This was the reason that MS signal of proteins without washing was observed (Figure $2 b$ ) although the $\mathrm{S} / \mathrm{N}$ ratio was relatively low. Following the first washing step of the PAA target (Figure 3c), most of the salt blocks disappeared, as indicated by the significant increase in the number and size of pores. Obviously, most of the discontinued salt blocks were removed by washing, resulting in the exposure of proteins adsorbed on the PAA surface for ionization. Therefore, MS spectra with a better $\mathrm{S} / \mathrm{N}$ ratio could be obtained. Although the washing process could remove not only the salt but also the proteins deposited on the PAA (as described in Figure 1), sufficient amounts of protein remaining on the PAA membrane as MALDI still produced strong signals. This result was in good agreement with that obtained with the infrared spectroscopic measurement. These results showed that use of PAA membrane as target was an excellent means to avoid the problems of high amount of salts in protein mixture analysis. 

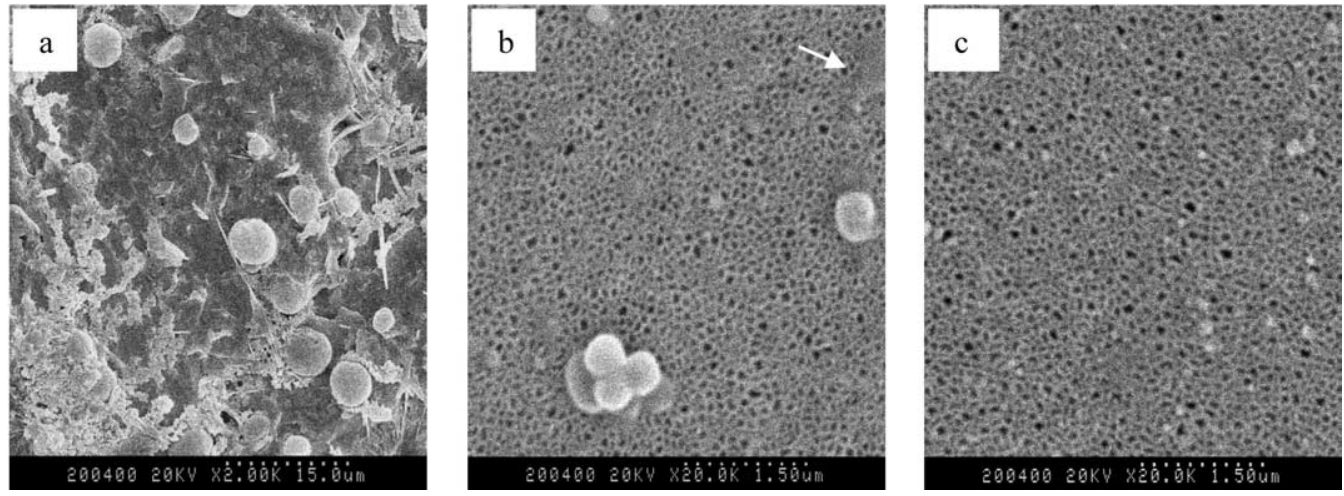

Figure 3. SEM images of a metallic target (a) and a PAA target (b) deposited with lysozyme sample $(200 \mathrm{pmol})$ dissolved in a solution of phosphate $(350 \mathrm{mM})$ buffer and acetonitrile with CHCA. Image (c) corresponds to the sample (b) washed with methanol and water (1:4, vol/vol) once.

\section{Conclusions}

We have first demonstrated the use of porous anodic alumina (PAA) membrane as sample support for MALDI-TOF MS analysis of proteins. The resulting spectral quality of proteins with standard sample preparation conditions is better than that of normal metal sample stages. Because of the affinity of PAA membrane toward biomolecules, as shown by FTIR measurement, the present technique allows us to introduce a desalting step before MS analysis. Therefore, high quality spectra of proteins on the PAA target can be achieved simply by a washing protocol. The present technique is promising for proteomics research.

\section{Acknowledgments}

This work was supported by grants from the National Natural Science Foundation of China (NSFC, 20125515; 20375016; 20299033; 20175034; 20475059) and the Chinese Academy of Science (KGCX2-SW-213-04).

\section{References}

1. Karas, M.; Hillenkamp, F. Laser Desorption Ionization of Proteins with Molecular Masses Exceeding 10,000 Daltons. Anal. Chem. 1988, 60, 2299-2301.

2. Tanaka, K.; Waki, H.; Ido, Y.; Akita, S.; Yoshida, Y.; Yoshida, T. Protein and Polymer Analyses up to $m / z$ 100,000 by Laser Ionization Time-of-flight Mass Spectrometry. Rapid Commun. Mass Spectrom. 1988, 2, 151-153.

3. Laugesen, S.; Roepstorff, P. Combination of Two Matrices Results in Improved Performance of MALDI MS for Peptide Mass Mapping and Protein Analysis. J. Am. Soc. Mass Spectrom. 2003, 14, 992-1002.

4. Fenselau, C. MALDI MS and Strategies for Protein Analysis. Anal. Chem. 1997, 69, 661A-665A.

5. Hillenkamp, F.; Karas, M.; Beavis, R.C.; Chait, B. T. MatrixAssisted Laser Desorption/Ionization Mass Spectrometry of Biopolymers. Anal. Chem. 1991, 63, 1193A-1203A

6. Chou, J. Z.; Kreek, M. J.; Chait, B. T. Matrix-Assisted Laser Desorption Mass Spectrometry of Biotransformation Products of Dynorphin A in Vitro. J. Am. Soc. Mass Spectrom. 1994, 5, $10-16$.

7. Warren, M. E.; Brockman, A. H.; Orlando, R. On-Probe SolidPhase Extraction/MALDI-MS Using Ion-Pairing Interactions for the Cleanup of Peptides and Proteins. Anal. Chem. 1998, 70, 3757-3761.

8. Brockman, A. H.; Dodd, B. S.; Orlando, R. A. Desalting Approach for MALDI-MS Using On-Probe Hydrophobic SelfAssembled Monolayers. Anal. Chem. 1997, 69, 4716-4720.

9. Winston, R. L.; Fitzgerald, M. C. Concentration and Desalting of Protein Samples for Mass Spectrometry Analysis. Anal. Biochem. 1998, 262, 83-85.

10. Xu, Y. D.; Watson, J. T.; Bruening, M. L. Patterned Monolayer/ Polymer Films for Analysis of Dilute or Salt-Contaminated Protein Samples by MALDI-MS. Anal. Chem. 2003, 75, 85-190.

11. Zhang, L.; Orlando, R. Solid-Phase Extraction/MALDI-MS: Extended Ion-Pairing Surfaces for the On-Target Cleanup of Protein Samples. Anal. Chem. 1999, 71, 4753-4757.

12. Mock, K. K.; Sutton, C. W.; Cottrell, J. S. Sample Immobilization Protocols for Matrix-Assisted Laser Desorption Mass Spectrometry. Rapid Commun. Mass Spectrom. 1992, 6, 233-238.

13. Xu, Y. D.; Bruening, M. L.; Watson, J. T. Nonspecific, On-Probe Cleanup Methods for MALDI-MS Samples. Mass Spectrom. Rev. 2003, 22, 429-440.

14. Oleschuk, R. D.; McComb, M. E.; Chow, A.; Ens, W.; Standing, K. G.; Perreault, H.; Marois, Y.; King, M. Characterization of Plasma Proteins Adsorbed onto Biomaterials by MALDITOFMS. Biomaterials 2000, 21, 1701-1710.

15. Vestling, M. M.; Fenselau, C. Poly(vinylidene difluoride) Membranes as the Interface Between Laser Desorption Mass Spectrometry, Gel Electrophoresis, and in Situ Proteolysis. Anal. Chem. 1994, 66, 471-477.

16. Kurien, B. T.; Matsumoto, H.; Scofield, R. H. Purification of Tryptic Peptides for Mass Spectrometry Using Polyvinylidene Fluoride Membrane. Ind. J. Biochem. Biophys. 2001, 38, 274-276.

17. Blackledge, J. A.; Alexander, A. J. Polyethylene Membrane as a Sample Support for Direct Matrix-Assisted Laser Desorption/Ionization Mass Spectrometric Analysis of High Mass Proteins. Anal. Chem. 1995, 67, 843-848.

18. Zaluzec, E. J.; Gage, D. A.; Allison, J.; Watson, J. T. Direct Matrix-Assisted Laser Desorption Ionization Mass Spectrometric Analysis of Proteins Immobilized on Nylon-Based Membranes. J. Am. Soc. Mass Specctrom. 1994, 5, 203-237.

19. McComb, M. E.; Oleschuk, R. D.; Manley, D. M.; Donald, L.; Chow, A.; O'Neil, J. D. J.; Ens, W.; Standing, K. G.; Perreault, H. Use of a Nonporous Polyurethane Membrane as a Sample Support for Matrix-Assisted Laser Desorption/Ionization Time-of-Flight Mass Spectrometry of Peptides and Proteins. Rapid Commun. Mass Spectrom. 1997, 11, 1716-1722.

20. Thompson, G. E.; Fumeaux, R. C.; Wood, G. C.; Richardson, J. A.; Goode, J. S. Nucleation and Growth of Porous Anodic Films on Aluminium. Nature 1978, 272, 433-435. 
21. Masuda, H.; Fukuda, K. Ordered Metal Nanohole Arrays Made by a Two-Step Replication of Honeycomb Structures of Alumina. Science 1995, 268, 1466-1468.

22. Yuan, J. H.; He, F. Y.; Sun, D. C.; Xia, X. H. A. Simple Method for Preparation of Through-Hole Porous Anodic Alumina Membrane. Chem. Mater. 2004, 16, 1841-1844.

23. Martin, C. R. Nanomaterials: A Membrane-Based Synthetic Approach. Science 1994, 266, 1961-1966.

24. Chio, J.; Sauer, G.; Nielsch, K.; Wehspohn, R. B.; Gosele, U. Hexagonally Arranged Monodisperse Silver Nanowires with Adjustable Diameter and High Aspect Ratio. Chem. Mater. 2003, 15, 776-779.

25. Reiss, B. D.; Freeman, R. G.; Walton, I. D.; Norton, S. M.; Smith, P. C.; Stonas, W. G.; Keating, C. D.; Natan, M. J. Electrochemical Synthesis and Optical Readout of Striped Metal Rods with Submicron Features. J. Eletroanal. Chem. 2002, 522, 95-103.
26. Yuan, J. H., Wang, K., Xia, X. H. Highly Ordered Platinum Nanotubles Arrays for Amperometric Glucose Sensing. Adv. Funct. Mater. 2005, 15, 803-809.

27. Lau, K. H. A.; Tan, L. S.; Tamada, K.; Sander, M. S.; Knoll, W. Highly Sensitive Detection of Processes Occurring Inside Nanoporous Anodic Alumina Templates: A Waveguide Optical Study. J. Phys. Chem. B 2004, 108, 10812-10818.

28. Djokic, S. S.; Burrell, R. E. Visual Detection of Protein Adsorption onto Electrochemically Oxidized Aluminum Surfaces. Biosens. Bioelectron. 1998, 13, 271-278.

29. Rahmelow, K.; Hubner, W. Secondary Structure Determination of Proteins in Aqueous Solution by Infrared Spectroscopy: A Comparison of Multivariate Data Analysis Methods. Anal. Biochem. 1996, 241, 5-13. 